\title{
Electromagnetic wave scattering by many small particles
}

\author{
A.G. Ramm \\ Mathematics Department, Kansas State University, Manhattan, KS 66506-2602, USA \\ Received 24 August 2006; accepted 3 September 2006 \\ Available online 18 September 2006 \\ Communicated by V.M. Agranovich
}

\begin{abstract}
Scattering of electromagnetic waves by many small particles of arbitrary shapes is reduced rigorously to solving linear algebraic system of equations bypassing the usual usage of integral equations. The matrix elements of this linear algebraic system have physical meaning. They are expressed in terms of the electric and magnetic polarizability tensors. Analytical formulas are given for calculation of these tensors with any desired accuracy for homogeneous bodies of arbitrary shapes. An idea to create a "smart" material by embedding many small particles in a given region is formulated.
\end{abstract}

(C) 2006 Elsevier B.V. All rights reserved.

MSC: 78A45; 78A48; 81V10

PACS: 02.00; 03.40.K; 03.80

Keywords: Electromagnetic wave scattering; Small particles of arbitrary shapes; Many-body scattering problem; Nanotechnology

\section{Introduction}

Wave scattering by small particles was studied by Rayleigh, starting in 1871. He understood that the main term in the field, scattered by a small particle, is the dipole radiation. A particle is small if $\lambda \gg a$, where $a$ is the characteristic dimension of the particle. The particle is assumed homogeneous with parameters $\varepsilon, \mu$ and $\sigma$. By $k=\frac{2 \pi}{\lambda}$ we denote the wave number in the medium, surrounding particles. There is a large literature on scattering by bodies, small in comparison with the wavelength (see [1,3-6] and references therein). Exact analytical solutions were found for spherical and ellipsoidal particles [4].

For particles of arbitrary shapes the author gave analytical formulas for S-matrix, which allow one to calculate this matrix with any desired accuracy [5]. Our aim in this Letter is to show that the many-body scattering problem for $N$ small particles in electromagnetic (EM) wave theory context can be rigorously reduced to solving linear algebraic systems (las) of equations bypassing the usual usage of integral equations.

The matrix elements of this las have physical meaning: they are expressed in terms of the polarizability tensors of small bodies. The decisive point is: the author has derived analytical formulas which allow one to calculate these tensors with any desired accuracy for bodies of arbitrary shapes. Our theory is developed in Section 2. The basic assumption is:

$$
a \ll \lambda \ll d,
$$

where $a$ is the characteristic dimension of the small particles and $d$ is the smallest distance between two distinct particles. In Section 2 the problem is formulated and a method of its solution is developed. Our theory in Section 2 uses some ideas, similar to the ideas in [2]. The principal difference between the results in [2] and in Section 2 is the following: the scattering coefficients in [2] are not known analytically, and should be calculated separately, while in our theory the analogs of these coefficients, the scattering

E-mail address: ramm@math.ksu.edu (A.G. Ramm). 
matrices $\mathcal{S}_{i}$, are given analytically, explicitly (see formulas (A.1), (A.5)-(A.7) in Appendix A). In [2] the isotropic point scatterers are considered, the scattering is assumed isotropic, and in Section 2 this assumption is not used. However, this difference is less important than the principal difference, mentioned above.

In Section 3 the scattering in a medium consisting of many small particles is discussed under assumption (1). At the end of this section we formulate an idea of creating a "smart" material nanotechnologically, by embedding many small particles in a given region in such a way that the resulting material would, for instance, have a desired radiation pattern.

In Section 4 the field near the boundary of small particles is discussed. In Appendix A some auxiliary results from [5] are given. These results are essential for our theory.

\section{Statement of the problem}

Consider the scattering of a monochromatic plane EM wave with frequency $\omega$ by $N$ small homogeneous particles $D_{i}, 1 \leqslant i \leqslant N$, with parameters $\varepsilon, \mu, \sigma$ (permittivity, magnetic permeability, conductivity) and Lipschitz boundaries $S_{i}$, placed in a medium with parameters $\varepsilon_{0}, \mu_{0}, \sigma_{0}=0$. Let $S:=\bigcup_{i=1}^{N} S_{i}, D:=\bigcup_{i=1}^{N} D_{i}$. The time-dependent factor $e^{-i \omega t}$ is omitted.

For simplicity let us assume that $\sigma$ is so large that condition $[\mathcal{N}, E]=0$ on $S$ holds, where $\mathcal{N}$ is the exterior unit normal to $S$ and $[\mathcal{N}, E]:=\mathcal{N} \times E$ is the cross product.

Our theory can be developed for impedance boundary condition $[\mathcal{N}, E]=\zeta[[\mathcal{N}, H], \mathcal{N}]$ as well, where $\zeta$ is the surface impedance, $\zeta=\left(\frac{\varepsilon^{\prime}}{\mu}\right)^{1 / 2}$, and $\varepsilon^{\prime}:=\varepsilon+\frac{i \sigma}{\omega}$. In this case the penetration depth $\delta$ of the EM field into the particle is given by the formula: $\delta=\sqrt{\frac{2}{\omega \sigma \mu}}$, and $\delta \ll a$ if $\omega \sigma$ is sufficiently large, $\mu$ being fixed, $E \sim \frac{\delta}{\lambda} H$ if $\frac{\sigma}{\omega} \gg \varepsilon$.

The governing equations are

$$
\begin{array}{ll}
\nabla \times E=i \omega \mu H, & \nabla \times H=-i \omega \varepsilon^{\prime} E \quad \text { in } D, \\
\nabla \times E=i \omega \mu_{0} H, & \nabla \times H=-i \omega \varepsilon_{0} E \quad \text { in } D^{\prime},
\end{array}
$$

$[\mathcal{N}, E]$ and $\varepsilon^{\prime} E \cdot \mathcal{N}$ are continuous across $S$,

$$
\left[\mathcal{N}, E^{\prime}\right]=-\left[\mathcal{N}, E_{0}\right] \quad \text { on } S, E=E_{0}+E^{\prime}, H=H_{0}+H^{\prime},
$$

where $E_{0}, H_{0}$ is the incident field, which satisfies (3) in the whole space, $E^{\prime}, H^{\prime}$ is the scattered field. Let

$$
\mathcal{U}:=\left(\begin{array}{c}
E \\
H
\end{array}\right)
$$

and $\mathcal{S}_{i}$ be the scattering matrix corresponding to a small particle $D_{i}$. This means that if an electromagnetic wave $\mathcal{U}$ is incident upon $D_{i}$, then the scattered field $\mathcal{U}^{\prime}$ in the far-field zone is

$$
\mathcal{U}^{\prime}=\frac{e^{i k r}}{k r} \mathcal{S}_{i} \mathcal{U}+o\left(\frac{1}{r}\right), \quad r=\left|x-x_{i}\right| \gg \lambda \gg a,
$$

where $x_{i}$ is a point in $D_{i}$, which can be chosen arbitrarily, and it does not matter which point is chosen since $D_{i}$ is small. Formula (7) is valid if the field $\mathcal{U}$ is practically homogeneous within the distances of order $a$. The foundation of our theory is an explicit formula for $\mathcal{S}_{i}^{(n)}$ for a small homogeneous body of arbitrary shape, which allows one to calculate $\mathcal{S}_{i}$ with any desired accuracy:

$$
\left|\mathcal{S}_{i}^{(n)}-\mathcal{S}_{i}\right|=O\left(q^{n}\right), \quad 0<q<1,
$$

where $q$ is a constant which depends only on the geometry of $\mathcal{S}_{i}$ and the material constants $\varepsilon_{i}, \mu_{i}, \sigma_{i}$. This formula is derived by the author [5, Chapter 7] and the results we use in this Letter are presented below, in Appendix A.

If $\mathcal{S}_{i}, 1 \leqslant i \leqslant N$ are known and assumption (1) holds, then the EM field at any point $x \in \mathbb{R}^{3}$, such that

$$
\min _{1 \leqslant i \leqslant N}\left|x-x_{i}\right| \geqslant d,
$$

can be calculated by the formula:

$$
\mathcal{U}(x)=\mathcal{U}_{0}(x)+\sum_{i=1}^{N} g\left(x, x_{i}\right) \mathcal{S}_{i} \mathcal{V}\left(x_{i}\right), \quad g(x, y)=\frac{e^{i k|x-y|}}{k|x-y|} .
$$

The vectors $\mathcal{V}\left(x_{i}\right)$ in (10) are unknown. The expression $g\left(x, x_{i}\right) \mathcal{S}_{i} \mathcal{V}\left(x_{i}\right)$ is the field, scattered by $i$ th particle, placed in the field $\mathcal{V}\left(x_{i}\right)$. If $\mathcal{V}\left(x_{i}\right)$ were known, then formula (10) would give the solution to the $N$-body EM wave scattering problem in the region (9).

If $x$ is near the boundary $\mathcal{S}_{j}$, then one gets:

$$
\mathcal{U}(x)=\mathcal{U}_{0}(x)+\sum_{i \neq j} g\left(x, x_{i}\right) \mathcal{S}_{i} \mathcal{V}\left(x_{i}\right)+\left(\begin{array}{c}
\nabla \times \int_{\mathcal{S}_{j}} g(x, s) \mathbf{j}(s) d s \\
\frac{1}{i \omega \mu} \nabla \times \nabla \times \int_{S_{j}} g(x, s) \mathbf{j}(s) d s
\end{array}\right) .
$$


In (11) the integrals represent the electric and magnetic fields generated by the $j$ th particle in an immediate neighborhood of this body, and $\mathbf{j}$ is an unknown tangential field on $\mathcal{S}_{j}$, representing the induced by $\mathcal{U}$ surface current.

If assumption (1) holds, one may consider the $j$ th particle as being placed in the homogeneous incident field

$$
\mathcal{U}_{0}\left(x_{j}\right)+\sum_{i \neq j} g\left(x_{j}, x_{i}\right) \mathcal{S}_{i} \mathcal{V}\left(x_{i}\right),
$$

and the corresponding scattered field is

$$
\frac{e^{i k\left|x-x_{j}\right|}}{k\left|x-x_{j}\right|} \mathcal{S}_{j}\left(\mathcal{U}_{0}\left(x_{j}\right)+\sum_{i \neq j} g\left(x_{j}, x_{i}\right) \mathcal{S}_{i} \mathcal{V}\left(x_{i}\right)\right) .
$$

Therefore we get a linear algebraic system of equations for the unknown $\mathcal{V}\left(x_{i}\right)$ in (10):

$$
\mathcal{V}\left(x_{j}\right)=\mathcal{U}_{0}\left(x_{j}\right)+\sum_{i \neq j} g\left(x_{j}, x_{i}\right) \mathcal{S}_{i} \mathcal{V}\left(x_{i}\right), \quad 1 \leqslant j \leqslant N .
$$

If $\mathcal{S}_{j}, 1 \leqslant j \leqslant N$, are known, then (14) is a linear system of $6 N$ equations for the $6 N$ unknowns $\mathcal{V}\left(x_{j}\right)$, since $\mathcal{V}\left(x_{j}\right)$ is a 6-component vector. System (14) can be solved efficiently by iterations, provided that its matrix is diagonally dominant. The matrix of system (14) is

$$
I-\sum_{i \neq j} \mathcal{S}_{i} g\left(x_{j}, x_{i}\right)
$$

where $I$ is the unit matrix in the 6-dimensional space of vectors with complex-valued coordinates and the norm of a vector is defined as follows:

$$
\|\mathcal{U}(x)\|:=\max _{1 \leqslant i \leqslant 6}\left|\mathcal{U}_{i}(x)\right|, \quad \mathcal{U}=\left(\begin{array}{c}
\mathcal{U}_{1} \\
\vdots \\
\mathcal{U}_{6}
\end{array}\right), \quad E=\left(\begin{array}{c}
\mathcal{U}_{1} \\
\mathcal{U}_{2} \\
\mathcal{U}_{3}
\end{array}\right), \quad H=\left(\begin{array}{c}
\mathcal{U}_{4} \\
\mathcal{U}_{5} \\
\mathcal{U}_{6}
\end{array}\right) .
$$

Matrix (15) is diagonally dominant if

$$
\left\|\sum_{i \neq j} \mathcal{S}_{i} g\left(x_{j}, x_{i}\right)\right\|<1 .
$$

Inequality (17) holds if

$$
\frac{1}{k d} \max _{1 \leqslant m \leqslant 6} \sum_{\ell=1}^{6} \sum_{i \neq j}\left|\left(\mathcal{S}_{i}\right)_{m \ell}\right|<1 .
$$

Here we have used the estimate of the norm of a matrix $A$, corresponding to the norm (16):

$$
\|A\|=\max _{1 \leqslant m \leqslant 6} \sum_{\ell=1}^{6}\left|A_{m \ell}\right| .
$$

Using formula (A.1) (see Appendix A) in (18) one gets $\left|\left(\mathcal{S}_{i}\right)_{m \ell} g\left(x_{j}, x_{i}\right)\right|=O\left(\frac{k^{3} V}{k d}\right)=O\left(\frac{k^{3} a^{3}}{k d}\right)<1$ if assumption (1) holds. For example, if $k a \leqslant 0.1, k d=20, N<100$, then the left-hand side of (18) is less than $\frac{1}{20} \times 10^{-3} \times 6(N-1) \leqslant 3 \times 10^{-2}<1$, so that condition (18) holds, and the system (14) can be efficiently solved by iterations:

$$
\begin{aligned}
& \mathcal{V}_{j}^{(n+1)}=\mathcal{U}_{0 j}+\sum_{i \neq j} g_{j i} \mathcal{S}_{i} \mathcal{V}_{i}^{(n)}, \quad \mathcal{U}_{0 j}:=\mathcal{U}_{0}\left(x_{j}\right), g_{j i}:=g\left(x_{j}, x_{i}\right), \\
& \mathcal{V}_{j}^{(0)}=\mathcal{U}_{0 j}, \quad \mathcal{V}_{i}^{(n)}:=\mathcal{V}^{(n)}\left(x_{i}\right) .
\end{aligned}
$$

This completes the discussion of the scattering EM waves by $N$ small particles. In this discussion the field on the surface of $i$ th particle was not calculated.

This field is not needed for calculating the vectors $\mathcal{U}\left(x_{j}\right)$ which define the total field by formula (10) at all points except immediate neighborhoods of the particles. See Section 4 for the discussion of the field in an immediate neighborhoods of the particles. 


\section{Scattering in a medium consisting of small particles}

If the number $N$ of particles is very large, say $N \sim 10^{23}$, so that one discusses the EM wave scattering in a medium consisting of small particles, then assumption (1) implies that the number of small particles per unit volume of the space is $O\left(\frac{1}{d^{3}}\right)$ and their total volume per unit volume of the space is $O\left(\frac{a^{3}}{d^{3}}\right) \rightarrow 0$ if $\frac{a}{d} \rightarrow 0$. Therefore the sum in (10) in this limit tends to zero if assumption (1) holds. Assumption (1) was essential to our arguments, so, if $\varepsilon, \sigma$ and $\omega$ are fixed, we arrive at the conclusion that scattering by $N$ small particles, under the assumptions (1) and in the $\operatorname{limit}_{N \rightarrow \infty} \frac{a}{d}=0$, is vanishing in this limit. However, the physical situation is dramatically different if we assume that $\varepsilon=\varepsilon(\omega)$ and $\sigma$ may depend in a suitable way on $a$ and $d$.

For example, assume that the small particles are identical spheres of radius $a, \sigma=0$. It is known that the polarizability tensor of a ball equals to

$$
\alpha_{i j}=3 \varepsilon_{0} \frac{\varepsilon-\varepsilon_{0}}{\varepsilon+2 \varepsilon_{0}} \delta_{i j}
$$

where $\delta_{i j}$ is the Kronecker symbol, i.e., the unit matrix. Recall that the polarizability tensor $\alpha_{i j}$ of a dielectric body $D$ with the dielectric constant $\varepsilon$, placed in a constant electric field $E$ in a homogeneous medium with the dielectric constant $\varepsilon_{0}$, is defined by the equation:

$$
P_{i}=\alpha_{i j} V \varepsilon_{0} E_{j}
$$

where $V$ is the volume of $D$, over the repeated indices summation is understood, $P$ is the induced dipole moment acquired by the body $D$ in the field $E$, and $P_{i}$ is the $i$ th component of vector $P$.

Let us assume that the following limit exists:

$$
\lim _{b \rightarrow 0} \lim _{\nu \rightarrow \infty} \frac{\sum_{D_{j} \in B(y, b)} \mathcal{S}_{j}}{\operatorname{Vol} B(y, b)}:=q(y, \beta), \quad \operatorname{Vol} B(y, b)=\frac{4}{3} \pi b^{3},
$$

where the limit in (21) is taken as the number $v$ of particles in the ball $B(y, b)$ of radius $b$ centered at a point $y$ in $D$ tends to infinity in such a way that assumption (1) holds and $\frac{a}{d} \rightarrow 0$, and $\beta$ is the scattering direction, $\beta=\frac{x-y}{|x-y|}$ if the scattering wave is directed from point $y$ to point $x$.

Eq. (10) in the limit

$$
N \rightarrow \infty, \quad \frac{a}{d} \rightarrow 0, \quad a \ll \lambda \ll d, k=\frac{2 \pi}{\lambda},
$$

yields an integral equation for $\mathcal{U}(x)$ :

$$
\mathcal{U}(x)=\mathcal{U}_{0}(x)+\int_{D} \frac{e^{i k|x-y|}}{k|x-y|} q(y, x) \mathcal{U}(y) d y .
$$

If the particles are identical spheres of radius $a$, we have

$$
v \sim \frac{4}{3} \pi b^{3} / d^{3}, \quad \text { and } \quad \alpha_{i j}=3 \varepsilon_{0} \frac{\varepsilon-\varepsilon_{0}}{\varepsilon+2 \varepsilon_{0}} \delta_{i j},
$$

so the nonzero limit (21) exists if the limit

$$
\lim _{a / d \rightarrow 0} \frac{a^{3}}{d^{3}} \frac{\varepsilon-\varepsilon_{0}}{\varepsilon+2 \varepsilon_{0}}:=w \neq 0
$$

exists. This limit exists and $w=\frac{\varepsilon-\varepsilon_{0}}{\kappa}$, if

$$
\varepsilon=-2 \varepsilon_{0}+\kappa \frac{a^{3}}{d^{3}}, \quad \kappa \neq 0,
$$

where $\kappa$ is a constant. There also exist materials with $\varepsilon=\varepsilon(\omega)<0$ and $\sigma \neq 0$ for which the limit (25) exists. For example, if $\varepsilon:=\varepsilon(\omega)+i \frac{\sigma}{\omega}, \sigma \neq 0$, and

$$
\varepsilon(\omega) \rightarrow-2 \varepsilon_{0}, \quad \frac{i \sigma}{\omega} \rightarrow \kappa_{1} \frac{a^{3}}{d^{3}} \quad \text { as } \omega \rightarrow \omega_{0}, \kappa_{1} \neq 0,
$$

where $\omega_{0}$ is some frequency and $\kappa_{1}$ is a constant, then the nonzero limit (24) exists and, therefore, the nonzero limit (21) also exists.

Physically, it is natural to assume that the self-consistent (effective) field in a medium, consisting of many $\left(N \sim 10^{23}\right)$ small particles, should not change if any single particle is removed from the medium, in spite of the fact that the exact field can be changed 
very much in an immediate neighborhood of the removed particle [7]. For instance, if the boundary condition on the surface $S_{i}$ of this particle was $[\mathcal{N}, E]=0$ then, after the particle is removed, $[\mathcal{N}, E] \neq 0$ on $S_{i}$, so the relative change is infinite.

If the limit (21) exists, then Eq. (23) is the equation for the self-consistent field in the medium consisting of many small particles.

Our result allows one to formulate an idea for creating nanotechnologically a "smart" material by embedding many small particles in a given bounded region. Such an idea was first proposed for acoustic wave scattering in [9], see also [10]. The idea can be briefly described as follows. If the limit (21) exists, then the integral equation (23) holds. If for $x$ in the far zone $q(y, \beta):=$ $q(y, x)=q(y)$, so that $q$ does not depend on $x$, then Eq. (23) is equivalent to the Schrödinger equation with potential $q$. The "smart" material, which is characterized by a desired scattering amplitude (radiation pattern), can be constructed by first solving the inverse problem of finding such a $q$ from the desired scattering amplitude, that is, by solving an inverse scattering problem, and then relating this $q$ to the density of distribution of the small particles, embedded in our region. If $N(y)$ is the number of the small particles per unit volume in a neighborhood of the point $y$, then $q(y)$ is proportional to $N(y)$ (see [9]). Thus, if $q$ is found, then $N(y)$ is known, and the small particles should be embedded with the density $N(y)$ in order that the resulting "smart" material would have the desired radiation pattern. Such is the situation in acoustic wave scattering by small bodies. The details are discussed in this case in $[9,10]$.

In EM wave scattering the potential $q$, in general, may depend on both variables, $y$ and $x$. This leads to a new inverse problem of finding such a $q=q(y, x)$ from the scattering data, an open problem currently. Therefore, it is of interest to find if there are physically reasonable conditions under which $q(y, x)=q(y)$, because the inverse scattering problem in this case has been solved by the author (see [11], Chapter 5).

\section{Equation for the field in an immediate neighborhood of a particle}

Formula (10) was derived for $x$ satisfying condition (9). If one is interested in the field near $S_{j}$, one has to find $\mathbf{j}$ in Eq. (11) and then use formula (11) to calculate the field at a point $x$ near $S_{j}$.

The electric field near $S_{j}$ is

$$
E(x)=E_{0}(x)+\sum_{i \neq j} E_{i}(x)+\nabla \times \int_{S_{j}} g(x, s) \mathbf{j}(s) d s,
$$

where the vector $E_{i}(x)$ is calculated as the first three components of the vector

$$
\mathcal{S}_{i} \mathcal{U}\left(x_{i}\right) g\left(x_{j}, x_{i}\right),
$$

see formula (11). The boundary condition $\left.[\mathcal{N}, E]\right|_{S_{j}}=0$ and formula (27) yield an equation for $\mathbf{j}$ :

$$
A \mathbf{j}+\mathbf{j}=-\frac{1}{2 \pi}\left[\mathcal{N}, E_{0}(s)+\sum_{i \neq j} E_{i}(s)\right], \quad s \in S_{j}, 1 \leqslant j \leqslant N,
$$

where

$$
A \mathbf{j}:=\int_{S_{j}}\left[\mathcal{N}(s),\left[\nabla_{s} \frac{e^{i k|s-t|}}{2 \pi k|s-t|}, \mathbf{j}(t)\right]\right] d t, \quad s \in S_{j} .
$$

Formula (28) is analogous to formula (1.27) in [5], being its generalization to the potentials of single layer with the density $\mathbf{j}$ which is a tangential to $S_{j}$ vector field.

Eq. (28) is a Fredholm-type integral equation if $\mathcal{S}_{j}$ is a sufficiently smooth surface. It allows one to find $\mathbf{j}$ uniquely if $k^{2}$ is not an eigenvalue of the homogeneous Maxwell's equation in $D$ with the boundary condition

$$
\left.[\mathcal{N}, E]\right|_{S}=0 .
$$

If $k^{2}$ is such an eigenvalue, then this integral equation can be modified so that the modified equation is uniquely solvable for $\mathbf{j}$ (see [8]).

If $\mathbf{j}$ is found, then formula (11) allows one to calculate $\mathcal{U}=\left(\begin{array}{c}E \\ H\end{array}\right)$ in a neighborhood of $S_{j}$ and on $S_{j}$.

\section{Appendix A}

In this appendix we collect some of the results from [5] used in Section 2.

The formula for the $\mathcal{S}_{E}$-matrix for EM wave-scattering by a single small body is [5, p. 114]:

$$
\mathcal{S}_{E}=\frac{k^{3} V}{4 \pi}\left(\begin{array}{cc}
\mu_{0} \beta_{11}+\alpha_{22} \cos \theta-\alpha_{32} \sin \theta & \alpha_{21} \cos \theta-\alpha_{31} \sin \theta-\mu_{0} \beta_{12} \\
\alpha_{12}-\mu_{0} \beta_{21} \cos \theta+\mu_{0} \beta_{31} \sin \theta & \alpha_{11}+\mu_{0} \beta_{22} \cos \theta-\mu_{0} \beta_{32} \sin \theta
\end{array}\right) .
$$


In (A.1) $\mathcal{S}_{E}$ differs by the factor $k$ from the $\mathcal{S}$-matrix in [5, p. 114] because we use the dimensionless function $\frac{e^{i k r}}{k r}$, while in [5] the function $\frac{e^{i k r}}{r}$ is used. The notations are the same as in [5]:

$\theta$ is the angle of scattering, $\alpha_{i j}=\alpha_{i j}\left(\gamma_{\varepsilon}\right)$ is the electric polarizability tensor, $\gamma_{\varepsilon}:=\frac{\varepsilon-\varepsilon_{0}}{\varepsilon+\varepsilon_{0}}, \beta_{i j}$ is the magnetic polarizability tensor (see (A.7)), $\beta_{i j}=\alpha_{i j}(-1)$ if $\mu=\mu_{0} . V$ is the volume of the small body, and $\mathcal{S}_{E}$ is defined by the formula for the scattered electric field $E^{\prime}$ in the far zone:

$$
E^{\prime}=\frac{e^{i k r}}{k r} \mathcal{S}_{E} E, \quad r:=|x|,
$$

where $E$ is the incident field at the point where the small body is located. If $E^{\prime}$ is known, then

$$
H^{\prime}=i \omega \mu_{0} \frac{e^{i k r}}{r} i k\left[x^{0}, E^{\prime}\right], \quad x^{0}=\frac{x}{|x|} .
$$

Thus, if one knows $\mathcal{S}_{E}$, then one can calculate the matrix $\mathcal{S}$ in (7).

The matrix $\mathcal{S}_{E}$ in (A.1) is calculated in the coordinate system in which the wave vector $\mathbf{k},|\mathbf{k}|=k$, of the incident wave and vector $\mathbf{k}^{\prime}$ of the scattered wave are lying in one plane, $\theta$ is the angle between $\mathbf{k}$ and $\mathbf{k}^{\prime}$, the $x^{\prime}$-axis coincides with the $x$-axis, $y^{\prime}$-axis makes an angle $\theta$ with $y$-axis, $z^{\prime}$-axis is directed along $\mathbf{k}^{\prime}$, and the origin 0 is the same for $x, y, z$ and $x^{\prime}, y^{\prime}, z^{\prime}$ coordinates. The origin lies inside the small body (particle), and the plane $y 0 z=y^{\prime} 0 z^{\prime}$ is called the scattering plane. One has $E=\left(E_{1}, E_{2}, 0\right)$ in $x y z$ coordinates, $E^{\prime}=\left(E_{1}^{\prime}, E_{2}^{\prime}, 0\right)$ in $x^{\prime} y^{\prime} z^{\prime}$ coordinates, and

$$
\mathcal{S}_{E}\left(\begin{array}{c}
E_{2} \\
E_{1}
\end{array}\right)=\left(\begin{array}{c}
E_{2}^{\prime} \\
E_{1}^{\prime}
\end{array}\right) \text {. }
$$

Let us give formulas (see [5, pp. 54-55]) for calculating the polarizability tensors $\alpha_{i j}(\gamma)$,

$$
\gamma:=\left(\varepsilon-\varepsilon_{0}\right)\left(\varepsilon+\varepsilon_{0}\right):=\gamma_{\varepsilon},
$$

of a homogeneous body $D$ with boundary $S$, volume $V$, and permittivity $\varepsilon$, placed in the medium with permittivity $\varepsilon_{0}$. We have

$$
\left|\alpha_{i j}^{(n)}(\gamma)-\alpha_{i j}(\gamma)\right|=O\left(q^{n}\right), \quad 0<q<1
$$

where the $n$th approximation $\alpha_{i j}^{(n)}\left(\gamma_{\varepsilon}\right)$ to $\alpha_{i j}\left(\gamma_{\varepsilon}\right)$ is given by the formulas:

$$
\alpha_{i j}^{(n)}=\frac{2}{V} \sum_{m=0}^{n} \frac{(-1)^{m}}{(2 \pi)^{m}} \frac{\gamma^{n+2}-\gamma^{m+1}}{\gamma-1} b_{i j}^{(m)}, \quad n>0,
$$

and

$$
\begin{aligned}
& b_{i j}^{(m)}:=\int_{S} \int_{S} d s d t \mathcal{N}_{i}(t) \mathcal{N}_{j}(s) \int_{S} \underbrace{\cdots}_{m-1} \int_{S} \frac{1}{r_{s t_{m-1}}} \psi\left(t_{1}, t\right) \psi\left(t_{2}, t_{1}\right) \cdots \psi\left(t_{m-1}, t_{m-2}\right) d t_{1} \cdots d t_{m-1}, \\
& \psi(t, s):=\frac{\partial}{\partial \mathcal{N}_{t}} \frac{1}{r_{s t}}, \quad r_{s t}:=|s-t| \\
& \alpha_{i j}^{(1)}\left(\gamma_{\varepsilon}\right)=\alpha\left(\gamma_{\varepsilon}+\gamma_{\varepsilon}^{2}\right) \delta_{i j}-\frac{\gamma_{\varepsilon}^{2}}{\pi V} b_{i j}^{(1)} .
\end{aligned}
$$

By $\mathcal{N}_{j}(s)$ we denote the $j$ th component of the exterior unit normal to $S$ at the point $s \in S$.

The magnetic polarizability tensor is:

$$
\beta_{i j}:=\alpha_{i j}(-1)+\alpha_{i j}\left(\gamma_{\mu}\right), \quad \gamma_{\mu}:=\frac{\mu-\mu_{0}}{\mu+\mu_{0}} .
$$

If $\mu=\mu_{0}$ then $\alpha_{i j}\left(\gamma_{\mu}\right)=0$.

The induced electrical dipole moment $P$ on the body $D$, placed in a homogeneous electrostatic field $E$, is

$$
P_{i}=\alpha_{i j}\left(\gamma_{\varepsilon}\right) V \varepsilon_{0} E_{j}
$$

Here and below summation is understood over the repeated indices.

The induced magnetic moment is

$$
M_{i}=\beta_{i j} V \mu_{0} H_{j}
$$

The first term in (A.7) is absent if the penetration depth $\delta$ of the magnetic field into $D$ is much larger than the size $a$ of $D$, and is present if $\delta \ll a$, for example, if the body $D$ is a very good conductor in which $H$ cannot penetrate. 
Finally, let us explain the remark below (A.2) about the $\mathcal{S}$-matrix defined in (7). Matrix $\mathcal{S}_{E}$ in (A.1) has been obtained in [5] from formula (7.95) in [5]. This matrix allows one to calculate the scattered field $E^{\prime}$ by formula (A.4). If $E^{\prime}$ is known, then the scattered field $H^{\prime}$ can be calculated from the Maxwell equation (3):

$$
H^{\prime}=\frac{\nabla \times E^{\prime}}{i \omega \mu_{0}}=\frac{e^{i k r}}{r} \sqrt{\frac{\varepsilon_{0}}{\mu_{0}}}\left[x^{0}, E^{\prime}\right]
$$

which is formula (A.3), because $k=\omega \sqrt{\varepsilon_{0} \mu_{0}}$. Thus, the knowledge of the matrix (A.1) allows one to calculate $\mathcal{S}$-matrix in (7).

Let us discuss the role of the assumption (1). We have

$$
g:=\frac{e^{i k|x-y|}}{k|x-y|}=\frac{e^{i k|x| \sqrt{1-\frac{2\left(x^{0}, y\right)}{|x|}+\frac{|y|^{2}}{|x|^{2}}}}}{k|x| \sqrt{1-\frac{2\left(x^{0}, y\right)}{|x|}+\frac{|y|^{2}}{|x|^{2}}}}, \quad x^{0}=\frac{x}{|x|} .
$$

If $|y| \ll|x|$, then

$$
g=\frac{e^{i k|x|}}{k|x|} e^{-i k\left(x^{0}, y\right)}\left(1+O\left(\frac{|y|}{|x|}\right)+O\left(\frac{k|y|^{2}}{|x|}\right)\right), \quad k|y| \ll 1,|y| \ll|x| .
$$

If

$$
|y| \leqslant a, \quad|x| \geqslant d, \quad d \gg a, \quad a \ll \lambda, \quad \lambda=\frac{2 \pi}{k},
$$

then

$$
O\left(\frac{|y|}{|x|}\right)+O\left(k|y| \frac{|y|}{|x|}\right) \ll 1 .
$$

Thus, if $|x| \geqslant d,|y| \leqslant a, a \ll d$ and $a \ll \lambda$, then

$$
g \approx \frac{e^{i k|x|}}{|x|} e^{-i k\left(x^{0}, y\right)}
$$

The assumption $d \gg \lambda$ is not used in these estimates. Therefore, on the wavelength $\lambda$ there can be many small particles as long as each of these particles is in the far zone with respect to all other particles. This was used in [7] in a study of the acoustic wave scattering in a medium consisting of many $\left(N \sim 10^{23}\right)$ small, acoustically soft, particles.

In EM scattering one has to use the relation

$$
\nabla_{x} g=\frac{e^{i k r}}{k r}\left(i k-\frac{1}{r}\right) \frac{x-y}{|x-y|}, \quad r=|x-y|,
$$

and in the far zone one neglects the term $\frac{1}{r}$ compared with $i k$. This can be done if

$$
k \gg \frac{1}{d}, \quad r \geqslant d,
$$

and these inequalities imply

$$
d \gg \lambda \text {. }
$$

That is why we have imposed assumption (1) when dealing with EM wave scattering.

\section{References}

[1] G. Dassios, R. Kleinman, Low Frequency Scattering, Clarendon, Oxford, 2000.

[2] L. Foldy, Phys. Rev. 67 (1945) 107.

[3] V. Hulst, Light Scattering by Small Particles, Dover, New York, 1961.

[4] L. Landau, E. Lifshitz, Electrodynamics of Continuous Media, Pergamon, Oxford, 1993.

[5] A.G. Ramm, Wave Scattering by Small Bodies of Arbitrary Shapes, World Scientific, Singapore, 2005

[6] A.G. Ramm, Electromagnetic wave scattering by small bodies of arbitrary shapes, in: V. Varadan (Ed.), Acoustic, Electromagnetic and Elastic Scattering-Focus on T-matrix Approach, Pergamon, New York, 1980, pp. 537-546.

[7] A.G. Ramm, Phys. Lett. A 312 (2003) 256.

[8] A.G. Ramm, Scattering by Obstacles, Reidel, Dordrecht, 1986.

[9] A.G. Ramm, Distribution of particles which produces a desired radiation pattern, Commun. Nonlinear Sci. Numer. Simulation, in press.

[10] A.G. Ramm, Phys. Lett. A 360 (2006) 22.

[11] A.G. Ramm, Inverse Problems, Springer, New York, 2005. 